\title{
Spring jumpers vs power jumpers: ankle joint behavior in elite wushu athletes and implications for performance and injury risk
}

\author{
L. Benouaich ${ }^{\mathrm{a}}$, P. Rouch ${ }^{\mathrm{a}}$, F. Natta ${ }^{\mathrm{b}}$ and P. Thoreux

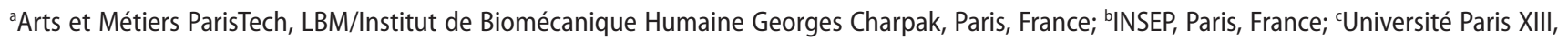 \\ Paris, France
}

KEYWORDS Jumping; stretch-shortening cycle; ankle; stiffness; acrobatics

\section{Introduction}

Wushu, better known as kungfu, is the modern athletic form of Chinese martial arts, and consists of performing a routine of martial arts movements as well as acrobatic jumps. Since those acrobatic jumps are performed on a hard floor, vertical jump height plays a major role in elite performance. Wushu coaches often mention two different types of athletes: spring jumpers and power jumpers. From this empirical point of view, spring jumpers seem to jump more easily.

As in many sports, wushu jumps are performed with a run up followed by a stance phase with eccentric and concentric phases, often referred to as a 'stretch-shortening cycle' (SSC). Vertical jump height, in this case, is the result of three main components acting during the concentric phase: muscle fiber shortening, coordination between joints and recoil of elastic energy stored during the eccentric phase. Whereas the squat jump exercise (SJ) is used to evaluate the first component, the drop jump (DJ) can be used as a standard exercise to analyze the whole SSC. The overall ability of an athlete to benefit from a previous eccentric phase can be evaluated by pre-stretch augmentation (PSA), defined as the ratio of DJ and SJ performances (Kubo et al. 2007).

Ankle behavior can be characterized by the evolution of joint torque, obtained with inverse dynamics, with respect to joint angle during the different phases of the support phase, with the slope being considered as overall joint stiffness.

The aim of this study was to characterize spring and power jumpers in elite wushu athletes in terms of PSA, ankle laxity, and overall ankle stiffness during the concentric phase. The influence of the chosen jumping strategies on performance and injury risk will be also discussed.

\section{Methods}

After standardized warm-up and maximal ankle dorsiflexion angle measures (Jones et al. 2005), 12 athletes of the French wushu team performed three repetitions of SJ and DJ, before performing 31 specific wushu jumps. Forty-two reflective markers were placed over skeletal landmarks. Kinematics were registered at $300 \mathrm{~Hz}$ with a 13 camera Vicon system and ground reaction forces with two AMTI force plates at $900 \mathrm{~Hz}$. Marker positions were filtered with an adaptive filter, and body segment inertial parameters were personalized. Ankle, knee, and hip joint reaction moments, calculated using inverse dynamics, as well as ankle flexion angle were expressed between 0 and 100\% of the support phase, and normalized by the body mass.

For SJ and DJ, performance was defined as the maximum height of the center of mass $(\mathrm{CoM})$ during the flight phase minus the CoM height in the static position. Data of the best performance for each subject in SJ and DJ were kept for analysis. PSA was defined as the ratio between the maximal performance in DJ and the maximal performance in SJ, minus 1. For the DJ, plantar flexion moments were plotted vs. ankle flexion angles, and two linear regressions were performed for the two halves of the concentric phase. The two regression slopes were defined as the ankle dorsiflexion stiffness in the first half of the concentric phase $\left(\mathrm{k}_{1}\right)$ and the ankle dorsiflexion stiffness in the second half of the concentric phase $\left(\mathrm{k}_{2}\right)$ in $\mathrm{Nm} /{ }^{\circ}$.

PSA results during DJ enabled the separation of the athletes into three groups of four subjects: power jumpers (low PSA), medium jumpers (medium PSA), and spring jumpers (high PSA).

Training experience and injury history were evaluated using a questionnaire. Wilcoxon tests were performed for statistical analysis, with spring jumpers as reference.

CONTACT L. leo.benouaich@gmail.com 

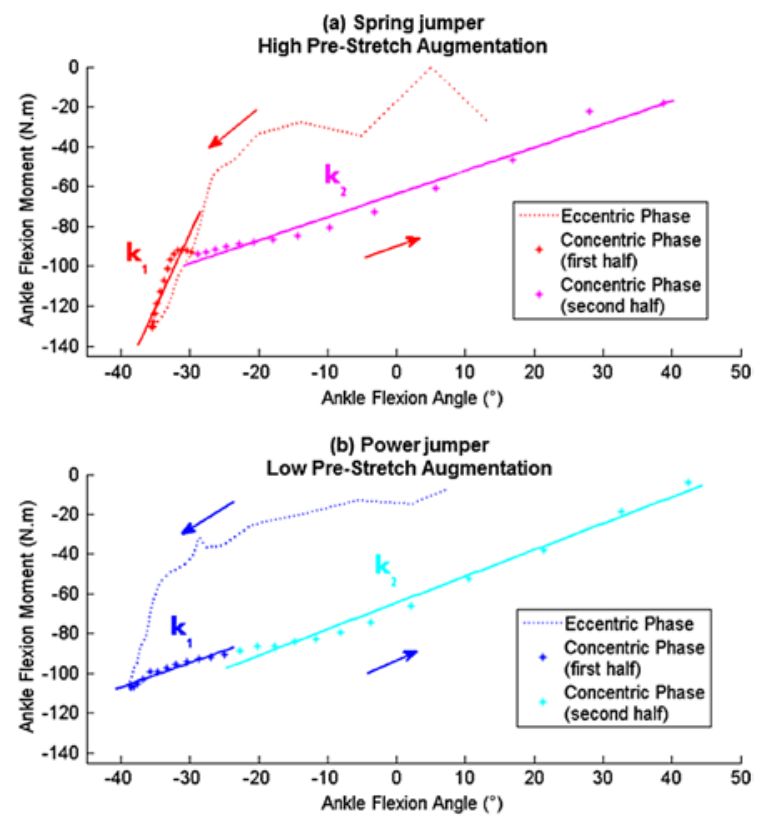

Figure 1. Ankle typical behavior in spring (a) and power jumpers (b) during the DJ stance phase. Linear regressions (full line) represent overall ankle stiffness during the first $\left(k_{1}\right)$ and the second half $\left(k_{2}\right)$ of the concentric phase.

\section{Results and discussion}

PSA was different between groups whereas performance, anthropometric data, and training experience were similar. In power and medium jumpers, $\mathrm{k}_{1}$ was lower than in spring jumpers $(p<0.05)$. Maximal dorsiflexion angle was lower in power jumpers than in spring jumpers $(p<0.05)$. Typical flexion moment vs. flexion angle curves for spring (a) and power (b) jumpers, and their respective $\mathrm{k}_{1}$ and $\mathrm{k}_{2}$ are presented in Figure 1. The bilinear evolution of ankle stiffness in spring jumpers can be interpreted as two different mechanisms predominating consecutively during the concentric phase. The first and higher slope could be mainly explained by the passive shortening of previously deformed articular structures, whereas the second and lower slope could be the effect of the active shortening of the triceps surae muscle fibers. In power jumpers, the second mechanism appears to be predominant.

The higher maximal dorsiflexion angle observed in power jumpers could explain the difficulty for these athletes in the storage and recovery during recoil of elastic energy at the ankle joint, and their choice of a predominant 'active strategy' during the concentric phase, in contrast to the 'combined active-passive strategy' used by spring jumpers.

Peak valgus reaction moments, in response to external adduction moments, were higher in spring and medium jumpers $(p<0.01)$. This parameter is often used as an index of knee osteoarthritis risk (Haim et al. 2012). In their injury history, the four spring jumpers were indeed more affected at knee level compared to power jumpers, but the small population does not allow statistical interpretation.

\section{Conclusions}

Concerning performance, absolute jump height was similar between power and spring jumpers, so their performance for acrobatic jump heights in competition would be similar. On the one hand, spring jumpers are the most efficient in terms of energy expenditure. The active strategy of power jumpers requires both high activation input by the central nervous system and high energy expenditure for muscle fiber shortening. These athletes may be more subject to a decrease in performance with fatigue. On the other hand, their larger ankle range of motion helps them in performing wushu compulsory low stances with heels on the ground.

It is difficult to know why athletes choose one strategy rather than the other. It could be linked with genetic abilities (laxity and muscle typology) as well as training habits. Power jumpers seem to practice more weight training compared to spring jumpers who are more used to specific plyometric training. It is also possible that some athletes, particularly in the medium group, do not choose the technique that best fits their capacities, explaining their lower overall performance.

For personalized training optimization and injury prevention, the athlete's jumping strategy should be taken into account, for instance, by avoiding repetitive jump training with power jumpers or by focusing on knee safety with spring jumpers.

\section{Acknowledgment}

The authors would like to thank the athletes for their involvement in the study.

\section{References}

Haim A, Rubin G, Rozen N, Goryachev Y, Wolf A. 2012. Reduction in knee adduction moment via non-invasive biomechanical training: a longitudinal gait analysis study. J Biomech. 45:41-45.

Jones R, Carter J, Moore P, Wills A. 2005. A study to determine the reliability of an ankle dorsiflexion weight-bearing device. Physiotherapy. 91:242-249.

Kubo K, Morimoto M, Komuro T, Yata H, Tsunoda N, Kanehisa H, Fukunaga T. 2007. Effects of plyometric and weight training on muscle-tendon complex and jump performance. Med Sci Sports Exercise. 39:1801-1810. 